\title{
Dohsa-hou intervention for reciprocal interpersonal interaction for a girl with Kabuki syndrome and autism spectrum disorder
}

\author{
Juri Kawano ${ }^{1}$ and Haruo Fujino ${ }^{2}$ \\ ${ }^{1}$ Oita University \\ ${ }^{2}$ Osaka University
}

April 2, 2021

\begin{abstract}
This case report illustrates an intervention using Dohsa-hou, a psychomotor therapy, for a girl with Kabuki syndrome and autism spectrum disorder. Although available evidence is limited, Dohsa-hou could be a treatment option for autism spectrum disorder associated with genetic disorders.
\end{abstract}

Dohsa-hou intervention for reciprocal interpersonal interaction for a girl with Kabuki syndrome and autism spectrum disorder

Juri Kawano, ${ }^{1}$ Haruo Fujino, ${ }^{1,2,3}$

${ }^{1}$ Department of Special Needs Education, Oita University, Oita, Japan

2 Department of Child Development, United Graduate School of Child Development, Osaka University, Suita, Japan

${ }^{3}$ Graduate School of Human Sciences, Osaka University, Suita, Japan

\section{Corresponding author}

Haruo Fujino

Department of Child Development, United Graduate School of Child Development, Osaka University

Address: 2-2 Yamadaoka, Suita, Japan, 5650871

Email: fjinoh@kokoro.med.osaka-u.ac.jp

Tel \& Fax: +81 668793863

\section{Ethical Publication Statement}

We confirm that we have read the Journal's position on issues involved in ethical publication and affirm that this report is consistent with those guidelines.

\section{Disclosure of Conflicts of Interest}

None of the authors has any conflict of interest to disclose.

\section{Abstract}

This case report illustrates an intervention using Dohsa-hou, a psychomotor therapy, for a girl with Kabuki syndrome and autism spectrum disorder. Although available evidence is limited, Dohsa-hou could be a treatment option for autism spectrum disorder associated with genetic disorders. 


\section{Key Clinical Message}

Although available evidence for psychosocial treatment for patients with Kabuki syndrome is limited, Dohsahou, a psychomotor therapy, could be a treatment option for autism spectrum disorder associated with the disorder.

Keywords : Kabuki syndrome, autism spectrum disorder, social interaction, Dohsa-hou, psychological intervention, interpersonal relations

\section{Introduction}

Kabuki syndrome is a rare genetic disease reported by Kuroki et al. and Niikawa et al. ${ }^{1,2}$ The frequency of the disease is estimated to be 1 in 34,000 people. ${ }^{3}$ The disease was defined as a malformation syndrome and reported five major symptoms: (1) characteristic facial features, (2) skeletal malformations, (3) dermatoglyphic abnormalities (various patterns on the surface of the skin), (4) mild to moderate intellectual disability, and (5) growth deficiency after birth. A majority of patients have pathogenic variant in KMT2D or KDM6A genes. However, there are a substantial number of patients who do not show pathogenic mutations in KMT2Dor KDM6A ${ }^{4-6}$ A recent international consensus proposed that a definitive diagnosis can be made based on a history of infantile hypotonia, developmental delay, and/or intellectual disability and typical dysmorphic features and/or pathogenetic variant inKMT2D or KDM6A. ${ }^{4}$

Most cases are characterized by mild to moderate intellectual disability, and a few patients have severe disabilities. ${ }^{7,8}$ There are several case reports of patients with Kabuki syndrome who exhibit features suggestive of autism spectrum disorders (ASD). ${ }^{9-12}$ ASD is primarily characterized by persistent deficits in social interaction and communication with others. Impairment in such abilities often leads to difficulties in development of interpersonal relationships. Therefore, development in communication with other people and experiencing support through interaction with others is an important issue. Generally, social communication was not impaired in most children and adolescents with Kabuki syndrome, although some patients had poor eye-contact and repetitive behavior. ${ }^{13,}{ }^{14}$ Such characteristics may differ from the typical behavior profile of ASD patients. In addition, insufficient information is currently available for psychological treatment for this specific population. ${ }^{15}$

There are several psychological treatments for children with ASD, such as social skills training. In Japan, interventions using Dohsa-hou have been implemented for children with ASD. Dohsa-hou is a psychotherapeutic approach developed in Japan, ${ }^{16-18}$ which focuses on body movement and psychomotor experience in individuals. ${ }^{19-21}$ There are several case reports presenting Dohsa-hou practice for children with ASD. ${ }^{22,}{ }^{23}$ In addition, Morisaki suggested three therapeutic goals in interacting with children with ASD through Dohsahou: experiencing relaxation, self-regulation of behavior, and sharing intention with others and realizing the presence of others. ${ }^{24}$ Joint attention and the sharing of intentions with others are essential aspects of development in social functioning and interaction in play among children. In addition, various studies have focused on the role of joint attention in the development of relationships with others. Joint attention is established through the interaction between the child and others and between the child and objects (such as toys) in a binary relationship, followed by the interaction between the child, objects, and others in a triadic relationship. ${ }^{25}$ This behavior is essential for the development of shared intentions to notice what others are paying attention to and has been focused on as a milestone of communication development necessary for subsequent social interaction skills. ${ }^{26,27}$

As suggested in the literature, such an interaction can lead to the development of social relationships and joint attention through the experience of sharing intention with body and movement, even with children in the pre-verbal stage. Currently, there is limited literature regarding psychotherapeutic interventions for children with Kabuki syndrome. ${ }^{15}$ However, previous reports related to autistic disorders in other populations may inform treatment considerations in Kabuki syndrome. Here, we report on a case study of a child with Kabuki syndrome and ASD, in which the changes in the child's behavior were examined through the intervention of Dohsa-hou. 


\section{Case Presentation}

\subsection{History}

At the beginning of the intervention, Sara was 7 years and 8 months old, and in the second grade of a special education school. When she was 3 years old, Kabuki syndrome was suspected based on the clinical signs, and she underwent genetic testing for KMT2D and KDM6Amutations, but no pathogenic mutations were detected by sequencing. Based on clinical manifestations and typical dysmorphic features of Kabuki syndrome, she was diagnosed with Kabuki syndrome, based on its cardinal features of the disorder.

Sara had severe intellectual disability and ASD, and her intellectual functioning was assessed using the Enjoji-developmental scale ${ }^{28}$ as severe intellectual delay (Developmental quotient=22). The subdomains were as follows: motor domain 36 , social 14 , and language 17 . She was nonverbal, and she could not pay attention to the objects only with verbal instructions. She had been receiving physical and occupational therapies once or twice a month at a medical center from 8 months to 6 years and 7 months.

\subsection{Intervention}

The Dohsa-hou intervention began in June 2018, and a total of 16 sessions were conducted for 6 months. The content of the Dohsa-hou intervention included interaction tasks, relaxation in the shoulders, back, and trunk, using hip joint in kneeling posture, and standing on the ground. ${ }^{16}$ The interaction task aimed to improve the interaction between Sara and the therapist by being aware of the therapist's intention. The therapist joined her hands with Sara, then moved the hand and arm upwards, with Sara matching the movement guided by the therapist.

After the intervention, playtime was set, and interactions with the therapist were video-recorded. Playtime was not set at sessions $1,3,6,8,12$, and 14 due to time constraints. From sessions 2-5, the child played freely, and in sessions $7-16$, she and the therapist played with the therapist's involvement.

Follow-up sessions were conducted 2 months after the end of 16 sessions. The intervention was delivered by a female therapist in training with the supervision of a certified clinical psychologist.

\subsection{Assessment}

Quality of shared intention in Dohsa-hou and play sessions:Shared intention in Dohsa-hou sessions was evaluated by the following six-point scale: Indifference (0), looking at the therapist but not trying to confirm the therapist's intentions (1), looking in the eyes when requesting for something (2), children looking at the therapist and the object (alternately) when the therapist points at the object (e.g., a body part) (3), making eye contact with the therapist in response to the action of the therapist (4), and making eye contact with the therapist spontaneously to show the therapist something (5).

Shared intention in play sessions were evaluated by the following six-point scale: Playing alone without paying attention to the therapist (0), paying attention to the therapist but playing alone (1), responding to encouragement from the therapist but returning to playing alone (2), playing together with the therapist but exhibiting limited motivation to share intentions (3), responding to encouragement from the therapist, sharing toys and playing, and playing together (4), the child seems to encourage the therapist to share their intentions (5).

To assess autistic symptoms, joint attention, and social adaptation, we used the Social Communication Questionnaire (SCQ), the Joint Attention Behavior Scale, and the Japanese version of the Vineland Adaptive Behavior Scale-II. These measures were administered at pre-intervention, session 10, session 16, and follow-up (2 months after the intervention).

Social Communication Questionnaire: The Social Communication Questionnaire (SCQ) is a screening tool to clarify the presence or absence of symptoms related to ASD, consisting of 40 items. ${ }^{29}$, 30 A higher score indicates higher autistic symptoms. It comprises three symptom domains (reciprocal interpersonal relationships, communication, and limited, repetitive, and stereotyped pattern of behaviors) that correspond to 
the Autism Diagnostic Interview-Revised (ADI-R), a clinical diagnostic instrument to assess ASD based on parent interviews. These domains were used to evaluate changes in autistic symptoms of the participant. Sara's overall score was 22 at pre-intervention, which is above the cutoff score for ASD screening.

Joint Attention Behavior Scale: This scale contains 17 items related to joint attention, which ${ }^{31}$ includes gazing, production and understanding of pointing, alternating gaze, understanding of others' emotions, and representations. The caregiver responded to the yes/no questions $(0 / 1)$. The higher the score, the more developed the behavior related to joint attention. She scored 0 at pre-intervention, suggesting profound impairment in joint attention.

Vineland Adaptive Behavior Scale-II: The Vineland Adaptive Behavior Scale-II (Vineland-II) is a semistructured interview that evaluates the adaptive behavior of individuals. It assesses the four domains of adaptive behavior: communication, daily living skills, social skills, and motor skills. ${ }^{32}$, 33 At pre-intervention, Sara's Vinland-II scores showed profound impairment in total score (Total $=21$ ) and all four domains ( communication $=22$, daily living skills $=20$, social skills $=20$, and motor skills $=20)$. The social skills domain (socialization) was repeatedly evaluated (intake, sessions 10 and 16, and follow-up), as this study focused on social relationships and behavior.

\subsection{Ethical Considerations}

This study was conducted in accordance with the recommendations of the Declaration of Helsinki of the World Medical Association, with written informed consent from the guardian of the participant. The study protocol was approved by the research ethics review board of the Faculty of Education at Oita University. The names and identifying details have been changed to protect the privacy of the participant.

\section{Results}

\subsection{The course of Dohsa-hou sessions}

During the intake session, there was little eye contact and little response to the therapist's name calling. There were many times when Sara seemed to reject the therapist offering her hand. At playtime, she did not respond to the therapist's words. She did not seem to respond to the therapist's attempts to play with her by showing her toys, and she also appeared to avoid the therapist's attempts. She did not make any requests nor maintained eye contact with the therapist and she played by herself.

In Sessions 1-5, in the interaction task, Sara sometimes tried to make hand movements with the therapist. She gradually turned her gaze toward the therapist and sometimes touched the therapist, indicating that she was trying to get involved with the therapist. At playtime, she made eye contact with the therapist a few times but turned away from the therapist who tried to engage her, and her response to the therapist's attempts were indifferent or negative. Sara showed little interest in the therapist and played by herself with a toy.

In Sessions 6-9, in the interaction task, when the therapist was assisting her, Sara tried to move her hands following the therapist and stared at the therapist more often. In addition, when the therapist looked at Sara's hand, she looked at her hand more often. She began to look at the therapist's face during the task and smiled several times. It seems that she was enjoying the task with the therapist. At this time, Sara often approached the therapist, held hands with the therapist, and waved her hands up and down as if she wanted to be involved with the therapist. At playtime in this period, she began to respond to the therapist's approach and smiled, although she sometimes started to play by herself. At that time, she began to reach for the toys and became more involved with the therapist. She began to share her attention with the therapist during play, for example, by looking at the therapist for confirmation.

In the interaction task in later sessions, Sara was able to move her body in accordance with the therapist's movements and intentions. When the therapist used words, such as "slowly" and "move with me" and moved her eyes, she moved following the therapist's movement. In addition, Sara began to show movements to the therapist as if she wanted to perform the interaction task with the therapist. In the task in kneeling posture, 
she was able to move her back in response to the therapist's instructions and was able to work calmly. At playtime in this period, her interest in the therapist's movements and actions was more pronounced. She sometimes sat in front of the therapist and looked at her expectantly. They often made eye contact, and when the therapist laughed, Sara often smiled as well. She shared her toy and played with the therapist steadily.

During the follow-up session, she was able to maintain eye contact with the therapist and continued to focus on each task. She concentrated on the therapist's gaze and movements and moved her arms in response to the therapist's intentions.

\subsection{Assessment}

Changes observed during the intervention are presented in Table 1. Although there were no major functional changes in the communication domain of ASD symptoms, there were improvements in reciprocal social interaction as assessed by the SCQ, interpersonal relationships as assessed by the Vineland-II, and play and leisure time by Session 16. No clear improvement was observed in joint attention or the other domains.

At the follow-up, Sara sometimes looked at her parents' eyes and faces and drew their attention to herself more than the pre-intervention period. She also smiled back when someone smiled at her, tried to join in interpersonal games, such as hand games, and responded favorably when other children approached her.

\section{Discussion}

In the present study, we described an intervention using Dohsa-hou for a girl with Kabuki syndrome associated with ASD and examined the changes during and after the intervention. Reciprocal interaction improved in her daily life, as well as in Dohsa-hou and play sessions. Regarding joint attention, changes were observed in the sharing of intentions during the sessions with the therapist, but no clear improvement was observed in daily life.

In the early stages of the Dohsa-hou intervention, Sara was not able to pay attention to her own body or the intention of the therapist in the interaction. As the session progressed, she became aware of the therapist's intentions and was able to share her intentions in the sessions. This process involved paying attention to her own body movements through physical experience and noticing the therapist's support (i.e., intentions) in the process. Through the process, there was a preceding change in the way she interacted with the therapist during the treatment sessions. Then, the changes in reciprocal relationships with others were observed in daily life. Shared intention and change in interactions through joint action coordination could be therapeutic factors that lead to reciprocal interpersonal relationships in ASD as suggested in recent literature. ${ }^{34,35}$ Therefore, interventions, such as Dohsa-hou and/or psychomotor therapy can be helpful for this population. ${ }^{12}$ As ASD are associated with certain cases of rare genetic disorders including Kabuki syndrome, ${ }^{36-38}$ such an approach would be a viable treatment option for these children with the disorders.

In this study, we only evaluated patient functioning; however, parental burden is also important for management of the families. ${ }^{39,40}$ Because no curative treatment is available for the disease, further research is required to establish adequate management practice for patients and families.

There are a few issues to be considered in this study. Kabuki syndrome is usually associated with less severe intellectual disability (i.e., mild to moderate) ${ }^{41}$ but the participants in this study had both severe intellectual disability and ASD. The process and quality of interaction may be affected by patient's functioning. Although joint attention was established with a specific person (i.e., the therapist), it did not generalize to other relationships. However, the Joint Attention Behavior Scale is a measure of disability and may not be appropriate for detecting therapeutic changes.

In conclusion, we described Dohsa-hou intervention for a girl with Kabuki syndrome, who has ASD and intellectual disability, that resulted in changes in her interaction with others and sharing of intentions. These changes may have been accompanied by changes in interaction with the therapist during the Dohsa-hou and play sessions. The Dohsa-hou could be a treatment option for ASD associated with genetic disorders. 


\section{Ethics statement}

This study was carried out in accordance with the ethical standards set forth in the 1964 Declaration of Helsinki and its later amendments, with written informed consent from the guardian of the participant. The protocol was approved by the Oita University Faculty of Education Research Ethics Committee (30-004). Names and identifying details have been removed to protect privacy of the participants.

\section{Competing interests}

The authors declare that they have no competing interests.

\section{Authors' contributions}

JK: developed the intervention, performed the intervention, interpreted the data, and wrote the first draft of the manuscript.

HF: developed the protocol, supervised the intervention, interpreted the data, and wrote the manuscript.

All the authors have read and approved the final manuscript.

\section{Acknowledgements}

The authors would like to thank the participant and her family.

\section{Reference}

1. Kuroki Y, Suzuki Y, Chyo H, et al. A new malformation syndrome of long palpebralfissures, large ears, depressed nasal tip, and skeletal anomalies associated with postnatal dwarfism and mental retardation. The Journal of Pediatrics 1981;99(4):570-73. doi: 10.1016/s0022-3476(81)80256-9

2. Niikawa N, Matsuura N, Fukushima Y, et al. Kabuki make-up syndrome: A syndrome of mentalretardation, unusual facies, large and protruding ears, and postnatal growth deficiency. The Journal of Pediatrics1981;99(4):565-69. doi: 10.1016/s0022-3476(81)80255-7

3. Niikawa N, Kuroki Y, Kajii T, et al. Kabuki make-up (Niikawa-Kuroki) syndrome: a study of 62 patients. Am J Med Genet1988;31(3):565-89. doi: 10.1002/ajmg.1320310312

4. Adam MP, Banka S, Bjornsson HT, et al. Kabuki syndrome: international consensus diagnostic criteria. J Med Genet 2019;56(2):89-95. doi: 10.1136/jmedgenet-2018-105625

5. Bogershausen N, Wollnik B. Unmasking Kabuki syndrome. Clin Genet 2013;83(3):201-11. doi: $10.1111 /$ cge. 12051

6. Murakami H, Tsurusaki Y, Enomoto K, et al. Update of the genotype and phenotype of KMT2D and KDM6A by genetic screening of 100 patients with clinically suspected Kabuki syndrome. Am J Med Genet A2020;182(10):2333-44. doi: 10.1002/ajmg.a.61793

7. Adam MP, Hudgins L. Kabuki syndrome: a review. Clin Genet2005;67(3):209-19. doi: 10.1111/j.13990004.2004.00348.x

8. Matsumoto N, Niikawa N. Kabuki make-up syndrome: a review. Am J Med Genet C Semin Med Genet 2003;117C(1):57-65. doi: 10.1002/ajmg.c.10020

9. Akin Sari B, Karaer K, Bodur S, et al. Case report: autistic disorder in Kabuki syndrome. J Autism Dev Disord 2008;38(1):198-201. doi: 10.1007/s10803-007-0433-x

10. Banka S, Lederer D, Benoit V, et al. Novel KDM6A (UTX) mutations and a clinical and molecular review of the X-linked Kabuki syndrome (KS2).Clin Genet 2015;87(3):252-8. doi: 10.1111/cge.12363

11. Ho HH, Eaves LC. Kabuki make-up (Niikawa-Kuroki) syndrome: cognitive abilities and autistic features. Dev Med Child Neurol1997;39(7):487-90. doi: 10.1111/j.1469-8749.1997.tb07470.x 
12. Parisi L, Di Filippo T, Roccella M. Autism spectrum disorder in Kabuki syndrome: clinical, diagnostic and rehabilitative aspects assessed through the presentation of three cases. Minerva Pediatr2015;67(4):36975 .

13. Mervis CB, Becerra AM, Rowe ML, et al. Intellectual abilities and adaptive behavior of children and adolescents with Kabuki syndrome: a preliminary study. Am J Med Genet A 2005;132A(3):248-55. doi: 10.1002/ajmg.a.30334

14. White SM, Thompson EM, Kidd A, et al. Growth, behavior, and clinical findings in 27 patients with Kabuki (Niikawa-Kuroki) syndrome. Am J Med Genet A 2004;127A(2):118-27. doi: 10.1002/ajmg.a.20674

15. Kasdon BD, Fox JE. Kabuki syndrome: diagnostic and treatment considerations. Ment Health Fam Med 2012;9(3):171-79.

16. Imura O, Chervenkova V. Introduction to Dohsa-hou: An integrated Japanese body-mind therapy: Osaka: Graduate School of Human Sciences, Osaka University. 2016.

17. Naruse G. Recent development of Dohsa-hou in Japan. Japanese Journal of Rehabilitation Psychology 1992;17-19:1-6.

18. Naruse G. Dohsa Ryoho [Dohsa-hou therapy]. Tokyo: Seishin Shobo 2000.

19. Fujino H. Effects of Dohsa-hou relaxation on body awareness and psychological distress. Jpn Psychol Res 2012;54(4):388-99.

20. Fujino H. Body awareness and mental health: A body psychotherapy case study. Body, Movement and Dance in Psychotherapy2016;11(4):249-62. doi: 10.1080/17432979.2016.1150882

21. Naruse G. The clinical Dohsa-hou as psychotherapy. Japanese Journal of Rehabilitation Psychology 1997;25(1):9-16.

22. Konnno Y. Principles and practice of the Dohsa-hou for autistic children. Jpn J Rehabil Psychol 1992;17-19:97-102.

23. Konnno Y. Establishing joint attention and communication in a child with autism through therapeutic touch, including a "sense of melting" experience: Dohsa method. Jpn J Spec Educ 1993;40(6):701-11. doi: 10.6033/tokkyou.40.701

24. Morisaki H. The significance of the mutual engagement using the body for children with autism: the theoretical consideration on a developmental point of view. Jpn J Rehabil Psychol2004;32(2):49-62.

25. Moore C, Dunham PJ. Joint attention: Its origins and role in development: Lawrence Erlbaum Associates, Inc. 1995.

26. Tomasello M, Carpenter M. Shared intentionality. Dev Sci2007;10(1):121-5. doi: 10.1111/j.14677687.2007.00573.x

27. Tomasello M, Carpenter M, Call J, et al. Understanding and sharing intentions: the origins of cultural cognition. Behav Brain Sci2005;28(5):675-91; discussion 91-735. doi: 10.1017/S0140525X05000129

28. Enjoji M. Infant Analytic Developmental Test of the Enjoji-Revised Edition. Tokyo, Japan: Keio University Press 1977.

29. Rutter M, Bailey A, Lord C. Social communication questionnaire: Japanese manual. Tokyo: Kanekoshobo 2013.

30. Rutter M, Bailey A, Lord C. Social communication questionnaire manual. Los Angeles, CA: Western Psychological Services 2003.

31. Kuroki M, Ohgami H. Standardization of joint attention behavior scale. Kyushu University Psychological Research 2003;4:203-13. 
32. Sparrow SS, Cicchetti D, Balla DA. Vineland Adaptive Behavior Scales-2nd edition manual. Minneapolis: NCS Pearson Inc. 2005.

33. Sparrow SS, Cicchetti DV, Balla DA. Japanese version of Vineland Adaptive Behavior Scales (2nd ed.). Tokyo: Nihon Bunka Kagakusha. 2014

34. Cerullo S, Fulceri F, Muratori F, et al. Acting with shared intentions: A systematic review on joint action coordination in autism spectrum disorder. Brain Cogn 2021;149:105693. doi: 10.1016/j.bandc.2021.105693

35. Trevisan DA, Enns JT, Birmingham E, et al. Action coordination during a real-world task: Evidence from children with and without autism spectrum disorder. Dev Psychopathol 2021;33(1):65-75. doi: $10.1017 /$ S0954579419001561

36. Bissell S, Wilde L, Richards C, et al. The behavioural phenotype of Potocki-Lupski syndrome: a cross-syndrome comparison. J Neurodev Disord 2018;10(1):2. doi: 10.1186/s11689-017-9221-x

37. Fujino H, Saito T, Matsumura T, et al. Autism spectrum disorders are prevalent among patients with dystrophinopathies. Neurol Sci2018;39(7):1279-82. doi: 10.1007/s10072-018-3341-2

38. Lane C, Milne E, Freeth M. Cognition and Behaviour in Sotos Syndrome: A Systematic Review. PLoS One 2016;11(2):e0149189. doi: 10.1371/journal.pone.0149189

39. Crane B, Alpert PT, Cyrkiel D, et al. Kabuki syndrome: a challenge for the primary care provider. $J$ Am Assoc Nurse Pract2013;25(10):522-6. doi: 10.1002/2327-6924.12044

40. Theodore-Oklota C, Egan S, Paulich M, et al. Caregiver-reported clinical characteristics and the burden associated with Kabuki syndrome.Am J Med Genet A 2020;182(7):1592-600. doi: 10.1002/ajmg.a.61584

41. Caciolo C, Alfieri P, Piccini G, et al. Neurobehavioral features in individuals with Kabuki syndrome. Mol Genet Genomic Med2018;6(3):322-31. doi: 10.1002/mgg3.348

Table 1. Assessment of ASD symptoms and socialization skills

\begin{tabular}{llll}
\hline Measure & Pre-intervention (Intake) & Session 10 & Post-intervention (Se \\
\hline SCQ & 22 & 21 & 16 \\
Reciprocal social interaction & 13 & 12 & 8 \\
communication & 8 & 8 & 8 \\
Restricted, repetitive, and stereotyped patterns of behavior & 0 & 0 & 0 \\
Joint Attention Behavior Scale & 0 & 1 & 2 \\
Vineland-II Socialization Domain & 4 & 4 & 5 \\
Interpersonal Relationships score (index) & $11(1)$ & $14(1)$ & $16(2)$ \\
Play and Leisure Time score (index) & $5(1)$ & $8(1)$ & $11(1)$ \\
Coping skills score (index) & $2(2)$ & $4(2)$ & $4(2)$ \\
\hline
\end{tabular}

SCQ, Social Communication Questionnaire; Vinland-II, Vineland Adaptive Behavior Scale-II

Figure legends

Figure 1. Quality of shared intention in Dohsa-hou and play sessions

FU: Follow-up 


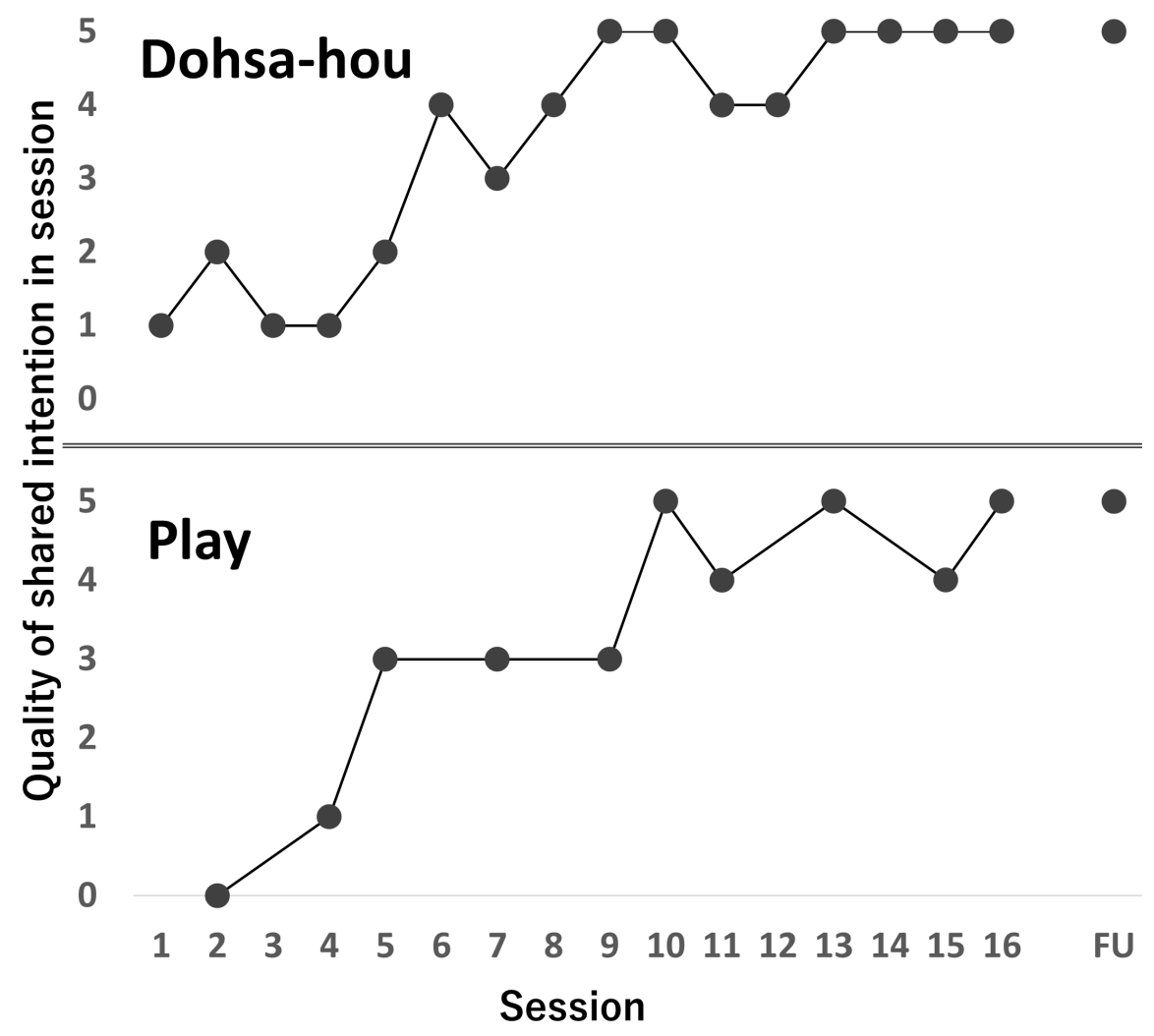

